\title{
Postpartum Headache: An Unexpected Manifestation of Posterior Reversible Encephalopathy Syndrome
}

\author{
Muhammad Effendi', Rashidi Ahmad', M. Zikri Ahmad', Harmy Mohamed Yusoff², Azhar bin Amir Hamzah³ \\ 'Department of Emergency, Hospital Universiti Sains Malaysia, Kubang Kerian, Kelantan, Malaysia \\ 2Department of Family Medicine, Hospital Universiti Sains Malaysia, Kubang Kerian, Kelantan, Malaysia \\ ${ }^{3}$ Department of Surgery, Hospital Universiti Sains Malaysia, Kubang Kerian, Kelantan, Malaysia
}

\begin{abstract}
Postpartum headache is described as complaints of headache and neck or shoulder pain in the first 6 weeks after delivery. The causes of postpartum headache include tension headache/migraine, preeclampsia/eclampsia, spinal headache, and cerebral pathology such as hemorrhage, thrombosis, or vasculopathy. We highlighted a case of a postpartum lady with a history of gestational hypertension who frequently attended out-patient facilities because of recurrent headaches. She had an episode of generalized tonic seizure that warranted visiting an emergency department. The findings of brain radio images were consistent with those of posterior reversible encephalopathy syndrome (PRES). We recommend a high index of suspicion of such a disease when assessing parturient women with recurrent headaches during the initial primary care visit. The clinical features, complications, and radio-imaging characteristics of PRES are presented. (Eurasian J Emerg Med 2016; 15: 108-10)
\end{abstract}

Keywords: Postpartum, headache, PRES

\section{Introduction}

Postpartum headache is one of the commonest morbidities experienced by approximately $38-40 \%$ of parturient women during puerperium. Most headaches occur during the first week following delivery (1).

The causes of postpartum headache could vary from simple primary causes of headache such as tension headache to lactation headache to the more serious secondary causes with intracranial pathologies (2).

Posterior reversible encephalopathy syndrome (PRES) is an infrequent diagnosis in day-to-day obstetric care, but it is known to be part of a spectrum of conditions associated with eclampsia (3). Early recognition and early aggressive treatment may prevent seizures and irreversible cognitive impairment.

The main purpose of highlighting this case is to increase awareness among primary care physicians regarding PRES and its specific management when managing patients with postpartum headache.

\section{Case Presentation}

A 26-year-old female, para 1, post her cesarean section delivery frequently visited the clinic because of recurrent episodes of dull aching headaches that were associated with photophobia. Headache intensity was attenuated by movement and relieved by taking paracetamol and resting. She denied blurring of vision or weakness in the limbs, and there was no associated nausea, vomiting, and epigastric pain. Furthermore, there was no history of a fall to suggest trauma, no syncope or other cardiovascular symptoms, and no fever to suggest an infectious etiology. She was on metoprolol $50 \mathrm{mg}$ twice daily for her gestational hypertension.

On day 6 postpartum, she had recurrent episodes of generalized tonic seizure with postictal drowsiness. There was no jerky movement of the limbs or urinary incontinence.

She was referred to the emergency department for stabilization and further management. On arrival, her GCS, BP, and HR were 15, $145 / 98 \mathrm{mmHg}$, and 104 beats per minute, respectively. A clinical examination revealed no abnormalities. She had moderate protein- 
uria and an elevated blood sugar level of $7.2 \mathrm{mmol} / \mathrm{L}$. Non-contrast computed tomography of the brain revealed the presence of white matter edema at the occipital and posterior parietal areas (Figure 1), which was suggestive of PRES. Subsequently, she underwent magnetic resonance imaging with findings of an abnormal signal intensity of white matter involving both parieto-occipital regions that appear iso-intense to gray matter on T1, hyper-intense on T2, and not suppressed on FLAIR, which confirmed the diagnosis (Figure 2).

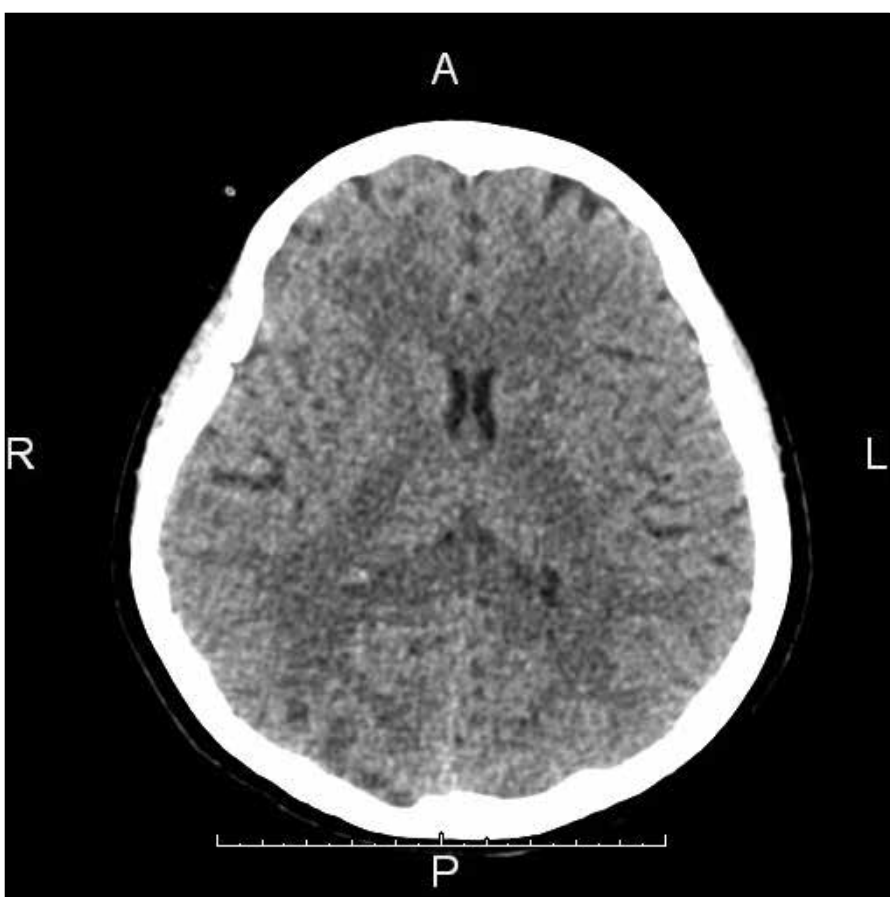

Figure 1. CT scan brain showing the parieto-occipital white matter edema

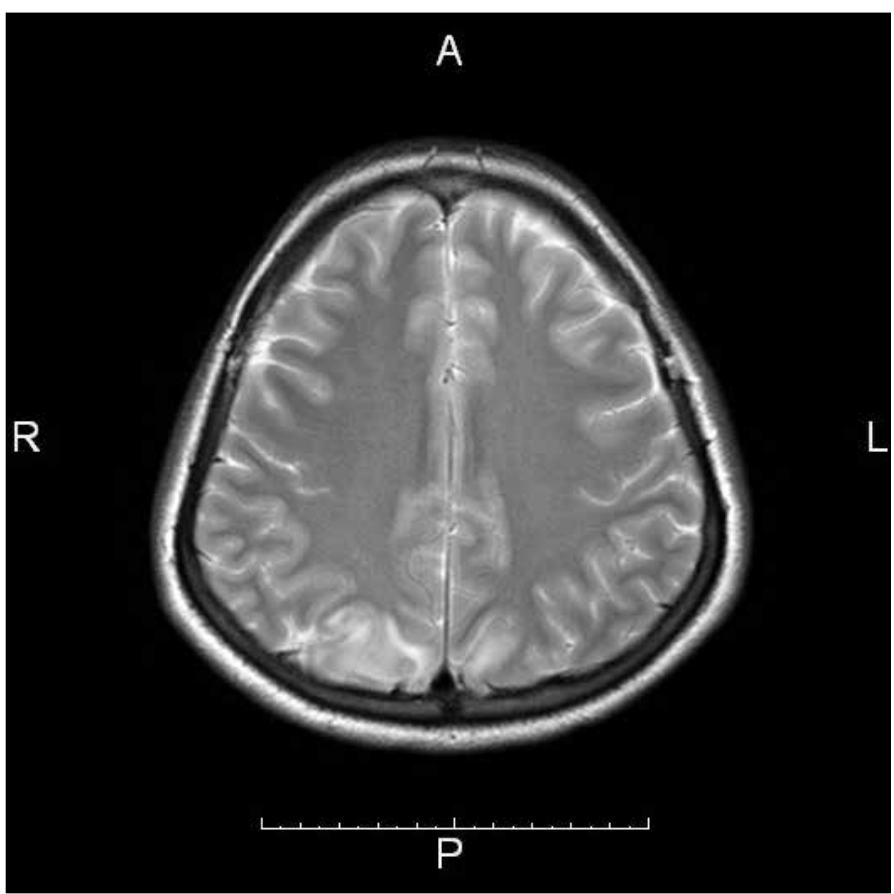

Figure 2. MRI of the brain showing an abnormal signal intensity of white matter involving both parietal-occipital regions that appear hyper-intense
She was started on an intravenous infusion of $4 \mathrm{~g}$ of magnesium sulfate. The dose of oral metoprolol was stepped up to $100 \mathrm{mg}$ twice daily. She was seizure-free afterward, and her blood pressure was within the normal range. Her higher mental functions were normal, and there was no neurological deficit. She was discharged on the fifth day after admission. She was completely healthy during a recent gynecological follow-up.

\section{Discussion}

Postpartum headache is a very common complaint in the primary care setting, and its causes could be benign or life threatening. The practicing physician should be aware of the various diagnostic and management modalities of this complaint. The mean onset of headache was 3.4 days (ranging from 2 to 32 days) after delivery. Tension headache/migraine headache was the most common cause $(47 \%)$ (4). However, other dangerous causes should be ruled out first.

PRES was quite recently recognized as a neuroradiological disorder. It is an infrequent diagnosis in day-to-day obstetric care, but it is known to be a part of a spectrum of conditions associated with eclampsia/preeclampsia (3). Apart from that, other risk factors for PRES include a history of malignant hypertension, fluid overload, sepsis, organ transplant recipients, renal failure, surgical patients, and malignancy with cytotoxic chemotherapy $(5,6)$. PRES is less common in people who live with chronic hypertension because they have adapted to the elevation in their blood pressure over time (6). PRES is characterized by headache, altered mental status, cortical blindness, and seizures. Persistent headaches lasting for more than $24 \mathrm{~h}$ after delivery warrant a re-evaluation of diagnosis. The cardinal systemic sign of PRES is an acute rise in the diastolic blood pressure, which can occur $24 \mathrm{~h}$ or more before other symptoms appear. In a limited primary care setting, any postpartum headache should be considered at risk; hence, further blood pressure monitoring and urine protein screening are considered to be mandatory. At this point, the assessment of airway, breathing, and circulation should have been performed with the availability of a good intravenous access. The next important step is the anticipation and management of potential catastrophic complications i.e., uncontrollable seizures and neurologic sequelae.

Although the name suggests that it is usually reversible, early diagnosis and treatment are essential because irreversible neurologic deficits or death may occur (3). Treatment involves lowering the mean arterial blood pressure to below $125 \mathrm{mmHg}$ and anticonvulsant therapy. The primary treatment for PRES is the appropriate pharmacological intervention for blood pressure control (mean arterial blood pressure should be less than $125 \mathrm{mmHg}$ ). Nicardipine and labetalol are the hypertensive drugs of choice. Nitroglycerine should be avoided because its vasodilator effects may attenuate cerebral edema (7). Other medications include analgesics for headaches and anticonvulsants for seizure prevention and treatment. For non-pregnant patients experiencing seizure, benzodiazepines (such as lorazepam or diazepam) are considered as first-line drugs, and they provide rapid onset and therapeutic benefits (8). Magnesium sulfate is considered to be the drug of choice for seizures in pregnancy (9). Uncontrollable seizure activity may require a higher level of care, intensive care monitoring, and support (8).

While not readily available to the primary physician, it is interesting to note that radio imaging characteristic of PRES is patchy white matter edema (6). The typical lesions are located in the pari- 
eto-occipital and posterior, frontal, cortical, and subcortical white matter, although the brain stem, basal ganglia, and cerebellum are less commonly involved (10). The mechanism of PRES is not yet clear. PRES may be associated with a failure in cerebral blood flow autoregulation combined with endothelial dysfunction (5). In this case, there is a possibility that the sudden rise in blood pressure disrupted the cerebral artery autoregulation; hence, there was a loss of cerebral blood flow control. Dilated arterioles may allow hyper perfusion, causing a breakdown in the bloodbrain barrier (5).

\section{Conclusion}

Though headaches in the postpartum period are usually benign, a high index of suspicion for dangerous causes of headache is considered necessary, particularly when the headache is severe, persistent, or associated with high blood pressure. Postpartum headache is one of the manifestations of PRES. Failure to recognize and institute appropriate management may lead to unwanted complications such as an unnecessary increase in maternal morbidity and mortality rates.

Peer-review: Externally peer-reviewed.

Conflict of Interest: No conflict of interest was declared by the authors.

Financial Disclosure: The authors declared that this study has received no financial support.

\section{References}

1. Pandya S. Postpartum headache: mandates correct clinical diagnosis and right intervention. JOACC 2011; 1: 55-6. [CrossRef]

2. Klein AM, Loder E. Postpartum headache. Int J Obstet Anesth 2010; 19: 422-30. [CrossRef]

3. Parisaei M, Derwig I, Yoon J, Erskine KJ, Jarman PR. Posterior reversible leukoencephalopathy in a case of postpartum eclampsia. Am J Obstet Gynecol 2005; 193: 885-6. [CrossRef]

4. Stella CL, Jodicke CD, How HY, Harkness UF, Sibai BM. Postpartum headache: is your work-up complete? Am J Obstet Gynecol 2007; 196: 318. e1-7.

5. Vaughn C, Zhang L, Schiff D. Reversible posterior leukoencephalopathy syndrome in cancer. Curr Oncol Rep 2008; 10: 86-91. [CrossRef]

6. O'Hara McCoy $\mathrm{H}$. Posterior reversible encephalopathy syndrome: an emerging clinical entity in adult, pediatric, and obstetric critical care. J Am Acad Nurse Pract 2008; 20: 100-6. [CrossRef]

7. Hinchey J, Chaves C, Appignani B, Breen J, Pao L, Wang A, et al. A reversible posterior leukoencephalopathy syndrome. N Engl J Med 1996; 334 494-500. [CrossRef]

8. Servillo G, Striano P, Striano S, Tortora F, Boccella P, De Robertis E, et al Posterior reversible encephalopathy syndrome (PRES) in critically ill obstetric patients. Intensive Care Med 2003; 29: 2323-6. [CrossRef]

9. Duley L, Henderson-Smart DJ, Walker GJ, Chou D. Magnesium sulphate versus diazepam for eclampsia. Cochrane Database Syst Rev 2010; CD000127.

10. Casey SO, Sampaio RC, Michel E, Truwit CL. Posterior reversible encephalopathy syndrome: utility of fluid-attenuated inversion recovery MR imaging in the detection of cortical and subcortical lesions. AJNR Am J Neuroradiol 2000; 21: 1199-206. 